\title{
Effects of 31 FDA approved small-molecule kinase inhibitors on isolated rat liver mitochondria
}

\author{
Jun Zhang ${ }^{1} \cdot$ Alec Salminen ${ }^{1,2} \cdot$ Xi Yang $^{1} \cdot$ Yong Luo $^{1} \cdot$ Qiangen $_{\mathrm{Wu}^{3}} \cdot$ \\ Matthew White ${ }^{1} \cdot$ James Greenhaw $^{1} \cdot$ Lijun Ren $^{1} \cdot$ Matthew Bryant $^{3}$. \\ William Salminen ${ }^{4}$ Thomas Papoian $^{5} \cdot$ William Mattes $^{1} \cdot$ Qiang Shi $^{1}$
}

Received: 15 September 2016 / Accepted: 14 December 2016 / Published online: 28 December 2016

(c) The Author(s) 2016. This article is published with open access at Springerlink.com

\begin{abstract}
The FDA has approved 31 small-molecule kinase inhibitors (KIs) for human use as of November 2016, with six having black box warnings for hepatotoxicity (BBW-H) in product labeling. The precise mechanisms and risk factors for KI-induced hepatotoxicity are poorly understood. Here, the 31 KIs were tested in isolated rat liver mitochondria, an in vitro system recently proposed to be a useful tool to predict drug-induced hepatotoxicity in humans. The KIs were incubated with mitochondria or submitochondrial particles at concentrations ranging from therapeutic maximal blood concentrations (Cmax) levels to 100-fold Cmax
\end{abstract}

This article is not an official guidance or policy statement of the US Food and Drug Administration (FDA). No official support or endorsement by the US FDA is intended or should be inferred.

Electronic supplementary material The online version of this article (doi:10.1007/s00204-016-1918-1) contains supplementary material, which is available to authorized users.

Qiang Shi

qiang.shi@fda.hhs.gov

1 Division of Systems Biology, National Center for Toxicological Research, Food and Drug Administration, 3900 NCTR Road, Jefferson, AR 72079, USA

2 Biomedical Engineering 2016, University of Arkansas, Fayetteville, AR 72701, USA

3 Division of Biochemical Toxicology, National Center for Toxicological Research, Food and Drug Administration, 3900 NCTR Road, Jefferson, AR 72079, USA

4 ProNatural Brands LLC, 1174 Southwest 5th Avenue, Boca Raton, FL 33432, USA

5 Division of Cardiovascular and Renal Products, Office of New Drugs I, Center for Drug Evaluation and Research, Food and Drug Administration, 10903 New Hampshire Avenue, Silver Spring, MD 20993, USA levels. Ten endpoints were measured, including oxygen consumption rate, inner membrane potential, cytochrome c release, swelling, reactive oxygen species, and individual respiratory chain complex (I-V) activities. Of the $31 \mathrm{KIs}$ examined only three including sorafenib, regorafenib and pazopanib, all of which are hepatotoxic, caused significant mitochondrial toxicity at concentrations equal to the $\mathrm{Cmax}$, indicating that mitochondrial toxicity likely contributes to the pathogenesis of hepatotoxicity associated with these KIs. At concentrations equal to 100 -fold Cmax, 18 KIs were found to be toxic to mitochondria, and among six KIs with BBW-H, mitochondrial injury was induced by regorafenib, lapatinib, idelalisib, and pazopanib, but not ponatinib, or sunitinib. Mitochondrial liability at 100-fold Cmax had a positive predictive power (PPV) of $72 \%$ and negative predictive power (NPV) of $33 \%$ in predicting human KI hepatotoxicity as defined by product labeling, with the sensitivity and specificity being $62 \%$ and $44 \%$, respectively. Similar predictive power was obtained using the criterion of Cmax $\geq 1.1 \mu \mathrm{M}$ or daily dose $\geq 100 \mathrm{mg}$. Mitochondrial liability at 1-2.5-fold Cmax showed a 100\% PPV and specificity, though the NPV and sensitivity were $32 \%$ and $14 \%$, respectively. These data provide novel mechanistic insights into $\mathrm{KI}$ hepatotoxicity and indicate that mitochondrial toxicity at therapeutic levels can help identify hepatotoxic KIs.

Keywords Hepatotoxicity · Kinase inhibitor · Drug induced liver injury $\cdot$ Mitochondrion $\cdot$ Submitochondrial particles

\section{Introduction}

Drug-induced liver injury (DILI) is a major safety concern for patients, clinicians, pharmaceutical companies, and 
regulatory agencies. Nearly $50 \%$ of orally administered drugs on the market have been linked to DILI, though the causality is not always clear (Weng et al. 2015b). DILI is of particular importance for small-molecule kinase inhibitors (KIs), a group of recently developed drugs used to treat various types of cancer and other diseases such as idiopathic pulmonary fibrosis. They may be recommended for longterm usage or to be used until unacceptable toxicity such as DILI is observed. In the product labeling, almost $20 \%$ (6 out of 31) of FDA approved KIs have a black box warning, the strongest safety warnings issued by the FDA, due to DILI, and $71 \%$ (22 out of 31) have a "Warnings and Precautions" section for DILI. The mechanisms and risk factors for KI hepatotoxicity may include metabolic activation (Castellino et al. 2012; Teo et al. 2015), direct mitochondrial damage (Weng et al. 2015a), oxidative stress (Xue et al. 2012), inhibition of hepatic transporters (Feng et al. 2009), and genetic variations in drug metabolism enzymes (Sugiyama et al. 2015; Takimoto et al. 2013). However, previous studies only investigated a small number (less than 10 in total) of KIs, and the results from different groups cannot be compared directly, as different models and platforms were used. A comprehensive study involving all FDA approved KIs is needed to help better understand KI hepatotoxicity regarding its mechanism and prediction for DILI.

Mitochondrial damage has long been recognized as an important mechanism for DILI (Meyers et al. 1988). Recent studies have gone a step further and provided evidence that mitochondrial liability was predictive of a chemical's potential to induce DILI in humans (Aleo et al. 2014; Porceddu et al. 2012). However, few KIs have been examined regarding mitochondrial toxicity. In the present study, all 31 FDA approved KIs were tested in isolated rat liver mitochondria at concentrations normalized to blood levels at or above therapeutic doses in humans. Mitochondrial functions that were measured in the current study included: oxygen consumption rate, inner membrane potential (MMP), cytochrome c release which reflects outer membrane integrity, swelling, reactive oxygen species (ROS), and the five individual respiratory chain complex (RCC $\mathrm{I}-\mathrm{V})$ activities. The results suggest that direct mitochondrial toxicity may contribute to the mechanism of hepatotoxicity induced by some KIs, but the predictive power of KI-induced mitotoxicity for clinical hepatotoxicity is rather limited.

\section{Materials and methods}

\section{Chemicals and reagents}

KIs were obtained from three vendors including Medkoo Biosciences (Chapel Hill, NC), Selleck Chemicals
(Houston, TX), and LC laboratories (Woburn, MA). Rhodamine 123 was from Cayman Chemical (Ann Arbor, MI). Cytochrome c profiling ELISA kit was from purchased Abcam Inc (Cambridge, MA). The ROS probe 5-(and-6)chloromethyl-2', $7^{\prime}$-dichlorodihydrofluorescein diacetate, acetyl ester (CM-H2DCFDA) was purchased from Thermo Fisher Scientific Inc (Grand Island, NY). Dimethyl sulfoxide (DMSO), sodium dodecyl sulfate (SDS), and other chemicals were obtained from Sigma-Aldrich (St Louis, MO).

\section{Characterization of commercial KIs by HPLC-Mass spectrometry}

KI stock solutions were prepared in DMSO and further diluted to $1 \mu \mathrm{g} / \mathrm{ml}$ as working standards with acetonitrile containing $0.1 \%$ formic acid. The solutions were stored at $-20{ }^{\circ} \mathrm{C}$ until analysis. Ten $\mu 1$ of each KI solution was injected into a Waters e2695 Alliance HPLC System coupled with both a Waters 2998 Photodiode Array (PDA) Detector and an ACQUITY QDa Mass Detector. Each analyte was eluted on a Waters Atlantis T3 C18 column $(4.6 \times 150 \mathrm{~mm}, 5 \mu \mathrm{m})$ at $40{ }^{\circ} \mathrm{C}$ using an isocratic mobile phase composed of $20 \% 10 \mathrm{mM}$ ammonium formate and $80 \%$ acetonitrile both containing $0.1 \%$ formic acid at a flow rate of $0.5 \mathrm{ml} / \mathrm{min}$. The eluate was monitored for up to $10 \mathrm{~min}$ by the PDA and by mass spectrometry with the electrospray ion source operating in the positive ion mode (ESI+) using MS scans of 100-1000 Da.

\section{Animal care}

Sprague-Dawley rats (220-350 g) were obtained from the U.S. Food and Drug Administration National Center for Toxicological Research (NCTR) breeding colony. Animal care and experimental procedures were approved by the NCTR Institutional Animal Care and Use Committee in accordance with the National Institutes of Health (NIH) "Guide for the Care and Use of Laboratory Animals" (http://www.ncbi.nlm.nih.gov/books/NBK54050). Preliminary experiments showed that mitochondria from male and female rats responded similarly to KI treatment. Therefore, only male rats were used in subsequent studies.

\section{Isolation of rat liver mitochondria}

Rat liver mitochondria were prepared as described previously (Weng et al. 2015a). Rats were anesthetized by an intraperitoneal injection of Nembutal and the liver perfused with PBS to remove the blood. The liver was then cut into small pieces and homogenized using a Dounce type homogenizer in the ice-cold isolation buffer (IB) containing $200 \mathrm{mM}$ sucrose, $10 \mathrm{mM}$ Tris-MOPS, $1 \mathrm{mM}$ EGTA 
(pH 7.4). The sample was then centrifuged at $600 \mathrm{~g}$ for $10 \mathrm{~min}$. The supernatant was further centrifuged at $7500 \mathrm{~g}$ for $10 \mathrm{~min}$, and the pellets was washed once with the IB and centrifuged again at $7500 \mathrm{~g}$ for $10 \mathrm{~min}$ to collect mitochondria. The protein concentration was measured using the Bradford method (Kruger 1994). Before drug treatment, the protein concentration was adjusted to $1 \mathrm{mg} / \mathrm{ml}$ using appropriate buffer. The purity of mitochondria was ascertained by detecting four mitochondrion-specific proteins and the cytosolic protein glyceraldehyde-3-phosphate dehydrogenase (GAPDH) using Western blot (Gusdon et al. 2015). Briefly, mitochondrial and cytosolic fractions from four different preparations using four rats were diluted using the Laemmli protein loading buffer and subject to SDS-PAGE with 4-20\% gradient gels. The proteins were blotted to PVDF membranes and detected using antibodies against GAPDH, ATP5A, UQCRC2, SDHB, and NDUFB8.

\section{Drug treatment}

The KI stock solutions were prepared in DMSO and aliquoted in $0.5 \mathrm{ml}$ tubes before storing at $-20{ }^{\circ} \mathrm{C}$. The aliquoted stock solutions were kept for less than one month and each tube was used only once. The KIs were added to the mitochondria at 1:1000 dilutions. The final concentrations for the majority of KIs were 100, 50, 30, 20, 10, 5, 2.5, and 1-fold of Cmax as reported in the product labeling and drug approval packages at the official FDA website (https://www.accessdata.fda.gov/scripts/cder/drugsatfda). The Cmax data were from clinical trials which used FDA recommend dosages, route of administration, and duration. Several KIs were tested at lower concentrations because of the low solubility in DMSO or the tendency to precipitate when added to test buffers or the interference with the assays. The final concentration of DMSO for drug treatment was always $0.1 \%$, and control treatments were $0.1 \%$ DMSO.

\section{Mitochondrial oxygen consumption}

A published procedure was followed to measure mitochondrial oxygen consumption (Weng et al. 2015a). Briefly, mitochondria were incubated with the KIs for $5 \mathrm{~min}$, and then split evenly and loaded to the sample tubes of two Oxytherm systems (Hansatech Instruments Ltd), with one system used for measuring glutamate/malate driven respiration and the other succinate-driven respiration, both of which were defined as state 4 respiration. After $3 \mathrm{~min}$, ADP was added to measure the maximal oxygen consumption rate, which was defined as state 3 respiration. The buffer used for these experiments contained $125 \mathrm{mM} \mathrm{KCl}, 10 \mathrm{mM}$ Tris-MOPS, $1 \mathrm{mM} \mathrm{KH_{2 }} \mathrm{PO}_{4}, 10 \mu \mathrm{M}$ EGTA-Tris, pH7.4, and was referred to as respiration buffer (RB). The oxygen consumption rate of DMSO-treated samples (controls) was set as 1 .

\section{Measurement of MMP}

MMP was measured using Rhodamine 123 as described in a previous report (Buron et al. 2010). Briefly, mitochondria were suspended in RB supplemented with $5 \mathrm{mM}$ succinic acid, $2 \mu \mathrm{M}$ rotenone and $10 \mu \mathrm{M}$ Rhodamine 123, and then KIs were added. The fluorescence (excitation $485 \mathrm{~nm}$, emission $535 \mathrm{~nm}$ ) was recorded every $1 \mathrm{~min}$ for $30 \mathrm{~min}$ using a Synergy 2 Multi-Mode microplate reader form BioTek (Winooski, VT).

\section{Mitochondrial ROS production}

ROS was measured using the fluorescence probe CMH2DCFDA (Mattiasson 2004). Briefly, freshly isolated mitochondria were diluted to $1 \mathrm{mg} / \mathrm{ml}$ using IB buffer containing $4 \mu \mathrm{M}$ CM-H2DCFDA. After $30 \mathrm{~min}$, the samples were centrifuged at $7500 \mathrm{~g}$ for $10 \mathrm{~min}$ and the resulting pellet re-suspended in a buffer containing $150 \mathrm{mM} \mathrm{KCl}$, $5 \mathrm{mM} \mathrm{KH}{ }_{2} \mathrm{PO}_{4}, 5 \mathrm{mM}$ Tris, $10 \mu \mathrm{M} \mathrm{CaCl}_{2}, 2 \mu \mathrm{M}$ rotenone, and $5 \mathrm{mM}$ succinate ( $\mathrm{pH}$ 7.4). KIs were then added, and $100 \mu \mathrm{M} \mathrm{CaCl}_{2}$ was used as a positive control. The fluorescence (excitation $490 \mathrm{~nm}$, emission $530 \mathrm{~nm}$ ) was measured every 1 min for 30 min by using a Synergy 2 Multi-Mode microplate reader form BioTek (Winooski, VT). The signal from DMSO-treated samples was set as 1 .

\section{Activities of RCC I-V}

The activities of RCC I-V were determined using previously published procedures (Kirby et al. 2007; Weng et al. 2014). Submitochondrial particles were prepared by subjecting the intact mitochondria to three successive freezethaw cycles (Kirby et al. 2007). KIs were incubated with submitochondrial particles $(1 \mathrm{mg} / \mathrm{ml})$ for $15 \mathrm{~min}$ and then RCC I-V activities measured. The final KI concentrations ranged from 1-100 fold Cmax. The activities of DMSOtreated samples (controls) were set as 1. RCC I activity was measured using a buffer containing $25 \mathrm{mM}$ potassium phosphate, $5 \mathrm{mM} \mathrm{MgCl}_{2}, \mathrm{pH} 7.2,0.25 \%$ bovine serum albu$\min$ (BSA), $0.13 \mathrm{mM} \beta$-Nicotinamide adenine dinucleotide, reduced dipotassium salt (NADH), $2 \mathrm{mM}$ potassium cyanide $(\mathrm{KCN}), 2 \mu \mathrm{g} / \mathrm{ml}$ antimycin $\mathrm{A}$ and $65 \mu \mathrm{M}$ ubiquinone 1 , which was supplemented with or without $2 \mu \mathrm{g} /$ $\mathrm{ml}$ rotenone. The difference between the activity from rotenone-containing buffer and rotenone-absent buffer was considered as RCC I activity. RCC II activity was measured in a buffer containing $25 \mathrm{mM}$ potassium phosphate, $5 \mathrm{mM} \mathrm{MgCl}_{2}, \mathrm{pH} 7.2,20 \mathrm{mM}$ sodium succinate, $50 \mu \mathrm{M}$ 
Table 131 KIs tested in the present study

\begin{tabular}{|c|c|c|c|c|c|}
\hline Drug name & $\begin{array}{l}\text { Approval date as a new } \\
\text { molecular entity }\end{array}$ & $\operatorname{Cmax}(\mu \mathrm{M})$ & $\begin{array}{l}\text { Recommended daily dose } \\
\text { (mg/day) }\end{array}$ & $\begin{array}{l}\text { DILI potential (based } \\
\text { on labeling) }\end{array}$ & labeling for DILI \\
\hline Imatinib & 10-May-01 & 2.00 & 600 & 1 & W\&P: fatal DILI \\
\hline Gefitinib & 5-May-03 & 0.27 & 250 & 1 & W\&P: fatal DILI \\
\hline Erlotinib & 18-Nov-04 & 3.44 & 150 & 1 & W\&P: fatal DILI \\
\hline Sorafenib & 20-Dec-05 & 4.30 & 400 & 1 & W\&P: fatal DILI \\
\hline Sunitinib & 26-Jan-06 & 0.12 & 44 & 1 & BBW: fatal DILI \\
\hline Dasatinib & 28-Jun-06 & 0.18 & 120 & 0 & NA \\
\hline Lapatinib & 13-Mar-07 & 4.18 & 1375 & 1 & BBW: fatal DILI \\
\hline Nilotinib & 29-Oct-07 & 4.27 & 700 & 1 & 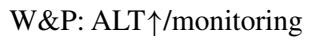 \\
\hline Pazopanib & 19-Oct-09 & 132.80 & 800 & 1 & BBW: fatal DILI \\
\hline Vandetanib & 6-Apr-11 & 1.80 & 300 & 0 & NA \\
\hline Vemurafenib & 17-Aug-11 & 0.13 & 960 & 1 & $\begin{array}{l}\text { W\&P: hepatic impairment/ } \\
\text { ALT monitoring }\end{array}$ \\
\hline Crizotinib & 26-Aug-11 & 0.91 & 500 & 1 & W\&P: fatal DILI \\
\hline Ruxolitinib & 16-Nov-11 & 1.20 & 25 & 0 & NA \\
\hline Axitinib & 27-Jan-12 & 0.07 & 10 & 1 & 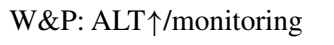 \\
\hline Bosutinib & 4-Sep-12 & 0.38 & 500 & 1 & 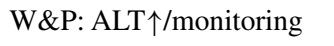 \\
\hline Regorafenib & 27-Sep-12 & 8.08 & 160 & 1 & BBW: fatal DILI \\
\hline Tofacitinib & 6-Nov-12 & 0.09 & 10 & 1 & 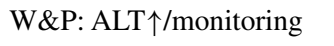 \\
\hline Cabozantinib & 29-Nov-12 & 2.58 & 100 & 0 & NA \\
\hline Ponatinib & 14-Dec-12 & 0.14 & 45 & 1 & BBW: fatal DILI \\
\hline Trametinib & 29-May-13 & 0.04 & 2 & 0 & NA \\
\hline Dabrafenib & 29-May-13 & 2.84 & 300 & 0 & NA \\
\hline Afatinib & 12-Jul-13 & 0.04 & 40 & 1 & 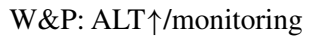 \\
\hline Ibrutinib & $12-F e b-14$ & 0.37 & 490 & 0 & NA \\
\hline Ceritinib & 29-Apr-14 & 1.81 & 750 & 1 & 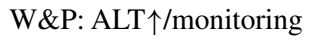 \\
\hline Idelalisib & 23-Jul-14 & 4.64 & 300 & 1 & BBW: fatal DILI \\
\hline Nintedanib & $15-O c t-14$ & 0.06 & 300 & 1 & 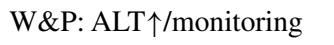 \\
\hline Palbociclib & 3-Feb-15 & 0.24 & 125 & 0 & NA \\
\hline Lenvatinib & $13-F e b-15$ & 0.68 & 24 & 1 & W\&P: fatal DILI \\
\hline Cobimetinib & 10-Nov-15 & 0.51 & 60 & 1 & W\&P: ALT $\uparrow /$ monitoring \\
\hline Osimertinib & 13-Nov-15 & 0.50 & 80 & 0 & NA \\
\hline Alectinib & 11-Dec-15 & 1.37 & 1200 & 1 & 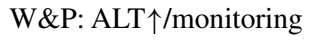 \\
\hline
\end{tabular}

$W \& P$ warnings and precautions, $B B W$ black box warnings, $A L T$ alanine aminotransferase, $N A$ not available. The 31 drugs are all FDA approved KIs for human use. Definition of DILI potential: 1, BBW and/or W\&P indicated that the drug cased DILI; 0, no DILI information available in the labeling. The six KIs with a BBW for hepatotoxicity are highlighted in red

2,6-dichloroindophenol sodium salt hydrate, $2 \mathrm{mM} \mathrm{KCN}$, $2 \mu \mathrm{g} / \mathrm{ml}$ antimycin A, $2 \mu \mathrm{g} / \mathrm{ml}$ rotenone, and $65 \mu \mathrm{M}$ ubiquinone 1 . RCC III activity was determined with a buffer containing $25 \mathrm{mM}$ potassium phosphate, $\mathrm{pH} 7.2,1 \mathrm{mM}$ n-dodecyl- $\beta$-D-maltoside, $1 \mathrm{mM} \mathrm{KCN,} 1 \mu \mathrm{g} / \mathrm{ml}$ rotenone, $100 \mu \mathrm{M}$ reduced-decylubiquinone, $15 \mu \mathrm{M}$ oxidized cytochrome c, and $0.1 \%$ BSA. RCC IV activity was measured using a buffer containing $25 \mathrm{mM}$ potassium phosphate, $\mathrm{pH}$ 7.2, $1 \mathrm{mM}$ n-dodecyl- $\beta$-D-maltoside, $15 \mu \mathrm{M}$ reduced cytochrome $\mathrm{c}$. RCC V was measured with a buffer containing $40 \mathrm{mM}$ Tris, $10 \mathrm{mM}$ EGTA, pH 8.0, $0.2 \mathrm{mM}$
NADH, $2.5 \mathrm{mM}$ phospho(enol)pyruvic acid monopotassium salt, $2.5 \mu \mathrm{g} / \mathrm{ml}$ antimycin A, $5 \mathrm{mM} \mathrm{MgCl}{ }_{2}, 5 \mathrm{U} / \mathrm{ml}$ lactate dehydrogenase, $5 \mathrm{U} / \mathrm{ml}$ pyruvate kinase, and $2.5 \mathrm{mM}$ ATP, which was supplemented with or without $2 \mu \mathrm{g} / \mathrm{ml}$ oligomycin A. The difference between the activity from oligomycin A-containing buffer and oligomycin A-absent buffer was considered as RCC V activity. For measuring RCC I/V and II activity, the changes in absorbance at 340 and $600 \mathrm{~nm}$, respectively, were determined every $1 \mathrm{~min}$ for 6 min. For measuring RCC III and RCC IV, the changes in absorbance at $550 \mathrm{~nm}$ were measured every $10 \mathrm{~s}$ for $2 \mathrm{~min}$. 
Table 2 Pharmacological targets and DILI potentials of FDA approved KIs

\begin{tabular}{|c|c|c|c|}
\hline Targets & DILI positive KIs & DILI negative KIs & $\begin{array}{l}\% \text { of DILI positive KIs (\# } \\
\text { of positive/negative KIs) }\end{array}$ \\
\hline ALK & Crizotinib; ceritinib; alectinib & None & $100 \%(3 / 0)$ \\
\hline PDGFR & $\begin{array}{l}\text { Imatinib; sorafenib; sunitinib*; nilotinib; pazopanib*; } \\
\text { axitinib; regorafenib*; ponatinib*; nintedanib; lenvatinib }\end{array}$ & Dasatinib & $91 \%(10 / 1)$ \\
\hline BCR-Abl & Imatinib; nilotinib; bosutinib; regorafenib*; ponatinib* & Dasatinib & $83 \%(5 / 1)$ \\
\hline VEGFR & $\begin{array}{l}\text { Sorafenib; sunitinib*; pazopanib*; axitinib; regorafenib*; } \\
\text { ponatinib*; nintedanib; lenvatinib }\end{array}$ & $\begin{array}{l}\text { Vandetanib; } \\
\text { cabozantinib }\end{array}$ & $80 \%(8 / 2)$ \\
\hline Flt3 & Sorafenib; sunitinib*; ponatinib*; nintedanib & Cabozantinib & $80 \%(4 / 1)$ \\
\hline Kit & $\begin{array}{l}\text { Imatinib; sorafenib; sunitinib*; } \\
\text { Pazopanib*; regorafenib*; ponatinib *; lenvatinib }\end{array}$ & Dasatinib; cabozantinib & $78 \%(7 / 2)$ \\
\hline RET & $\begin{array}{l}\text { Sorafenib; sunitinib*; regorafenib*; ponatinib*; len- } \\
\text { vatinib; alectinib }\end{array}$ & Vandetanib; cabozantinib & $75 \%(6 / 2)$ \\
\hline B-Raf & Sorafenib; vemurafenib; regorafenib* & Dabrafenib & $75 \%(3 / 1)$ \\
\hline EGFR & Gefitinib; erlotinib; lapatinib*; afatinib & Vandetanib; osimertinib & $67 \%(4 / 2)$ \\
\hline EphR & Regorafenib*; ponatinib* & Dasatinib; vandetanib & $50 \%(2 / 2)$ \\
\hline $\mathrm{Src}$ & Bosutinib; ponatinib* & Dasatinib; vandetanib & $50 \%(2 / 2)$ \\
\hline Tie2 & Regorafenib*; ponatinib* & Vandetanib; cabozantinib & $50 \%(2 / 2)$ \\
\hline MEK & Cobimetinib & Trametinib & $50 \%(1 / 1)$ \\
\hline JAK & Tofacitinib & Ruxolitinib & $50 \%(1 / 1)$ \\
\hline Lck & Pazopanib & Dasatinib & $50 \%(1 / 1)$ \\
\hline
\end{tabular}

* indicates the KIs with a black box warning for hepatotoxicity

\section{Mitochondrial swelling}

A previously published procedure was followed in detail for measuring mitochondrial swelling (Weng et al. 2015a).

\section{Cytochrome $c$ release assay}

Freshly isolated liver mitochondria $(1 \mathrm{mg} / \mathrm{ml}$ protein) were re-suspended in RB supplemented with $5 \mathrm{mM}$ succinic acid and $2 \mu \mathrm{M}$ rotenone, and then KIs were added, with DMSO used as a vehicle control and alamethicin as a positive control whose signal was set as 100 (Buron et al. 2010). The samples were incubated for $30 \mathrm{~min}$ with gentle shaking. After centrifugation $10,000 \mathrm{~g}$ for $10 \mathrm{~min}$, the supernatant and pellets were collected and kept at $-80{ }^{\circ} \mathrm{C}$. The cytochrome $\mathrm{c}$ level in the supernatant was determined using the cytochrome $\mathrm{c}$ profiling ELISA Kit from Abcam Inc. (Cambridge, MA) following the manufacturer's manual. As the antibody only reacts with denatured cytochrome c, it is of critical importance to incubate the supernatant with a buffer containing $1 \%$ SDS prior to measurement.

\section{Data analysis}

The means and standard deviations were obtained from at least three batches of mitochondria isolated from three different Sprague-Dawley male rats. The difference among treatment groups were analyzed by one-way or two-way
ANOVA followed by Dunnett's test using the software GraphPad Prism 6 (San Diego, California). A $p$ value less than 0.05 was considered statistically meaningful. The positive prediction value (PPV), negative prediction value (NPV), sensitivity, and specificity were calculated as previously described (Porceddu et al. 2012).

\section{Results}

\section{Overview of tested KIs}

All FDA approved small-molecule KIs were retrieved from the FDA official website at http://www.fda.gov/ Drugs/DevelopmentApprovalProcess/HowDrugsareDevelopedandApproved/DrugandBiologicApprovalReports/ NDAandBLAApprovalReports/ucm373420.htm. As of November 2016, the FDA has approved 31 KIs for human use. The Cmax, recommended daily dose, date of approval, and DILI potential of these drugs are summarized in Table 1. The recommended daily dose of these KIs ranged from 2 to $1375 \mathrm{mg}$, and the therapeutic Cmax ranged from 0.04 to $132.80 \mu \mathrm{M}$, a span of about three orders of magnitude, highlighting the importance of normalizing the test concentrations to $\mathrm{Cmax}$ for in vitro studies comparing KI toxicities. The chemical structure and molecular weight of KIs tested in this study were ascertained using mass spectrometry-based technology 

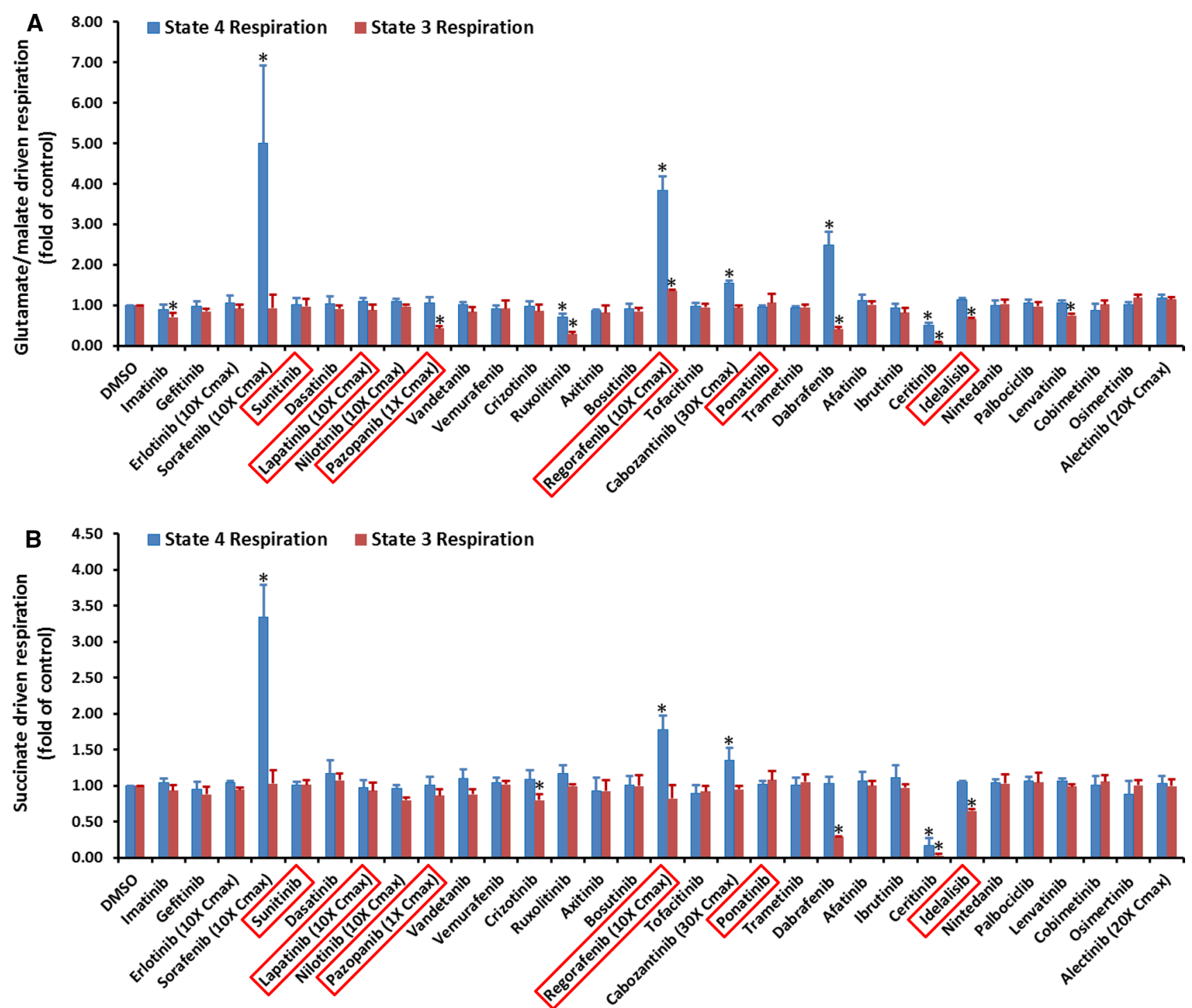

Fig. 1 KI effects on mitochondrial oxygen consumption. Rat liver mitochondria were incubated with KIs at 100 -fold Cmax or the maximal testable concentrations as indicated. The KIs in the $X$-axis were listed according to their approval date. The six KIs with a black box warning for hepatotoxicity were highlighted in a red box. The $Y$-axis represents the fold changes of oxygen consumption after KI treatment

(Supplementary Fig. 1). No quality issues were identified with these commercial KIs.

The known pharmacological targets of these KIs were collected from http://www.brimr.org/PKI/PKIs.htm and drug labeling from https://dailymed.nlm.nih.gov/dailymed/ index.cfm (Supplementary Table 1). The pharmacological targets associated with at least two approved KIs were listed in Table 2. The targets were listed according to the percentage of DILI positive KIs among each group. An interesting observation is that all 3 ALK inhibitors and 91\% (10 out of as compared to DMSO-treated samples. a, b Represent the oxygen consumption rate measured using glutamate/malate and succinate, respectively. Data are means and standard derivations from three separate experiments. ${ }^{*} p<0.05$ as compared to DMSO-treated samples (color figure online)

11) of PDGFR inhibitors are hepatotoxic. It is likely that KIs targeting these two pathways have exceptionally high risks of liver injury. As for several other targets, such as EphR, Sec and Tie2, only $50 \%$ of the corresponding KIs are hepatotoxic. This is in line with the observation that about 50\% (478 out of 975) of oral drugs have been associated with DILI (Weng et al. 2015b), indicating that targeting these kinase pathways is unlikely to cause additional DILI risks. However, definitive conclusions cannot be drawn at this time, as the number of KIs in each group is relatively small. 
Table 3 Dose response of KI effects on mitochondrial oxygen consumption

\begin{tabular}{|c|c|c|c|c|}
\hline \multirow{2}{*}{$\begin{array}{l}\text { Drugs } \\
\text { (fold of } \\
\text { Cmax) }\end{array}$} & \multicolumn{2}{|c|}{$\begin{array}{l}\text { Glutamate/malate driven } \\
\text { respiration }\end{array}$} & \multicolumn{2}{|c|}{ Succinate-driven respiration } \\
\hline & State 4 & State 3 & State 4 & State 3 \\
\hline \multicolumn{5}{|c|}{ Sorafenib } \\
\hline 10 & $5.00 \pm 1.92 *$ & $0.93 \pm 0.34$ & $3.34 \pm 0.45^{*}$ & $1.03 \pm 0.19$ \\
\hline 5 & $3.69 \pm 0.12 *$ & $1.34 \pm 0.04 *$ & $1.03 \pm 0.08$ & $0.40 \pm 0.01 *$ \\
\hline 2.5 & $4.92 \pm 0.09^{*}$ & $1.57 \pm 0.06^{*}$ & $1.43 \pm 0.06^{*}$ & $0.62 \pm 0.05^{*}$ \\
\hline 1 & $2.55 \pm 0.27^{*}$ & $1.15 \pm 0.09$ & $1.50 \pm 0.21^{*}$ & $1.00 \pm 0.10$ \\
\hline \multicolumn{5}{|c|}{ Regorafenib } \\
\hline 10 & $3.84 \pm 0.34^{*}$ & $1.36 \pm 0.03 *$ & $1.78 \pm 0.19^{*}$ & $0.82 \pm 0.19$ \\
\hline 5 & $3.82 \pm 0.72 *$ & $1.33 \pm 0.04 *$ & $1.69 \pm 0.09^{*}$ & $0.83 \pm 0.03$ \\
\hline 2.5 & $5.51 \pm 0.35^{*}$ & $2.51 \pm 0.04^{*}$ & $3.59 \pm 0.14^{*}$ & $0.84 \pm 0.04$ \\
\hline 1 & $7.45 \pm 0.17^{*}$ & $2.33 \pm 0.06^{*}$ & $2.95 \pm 0.08^{*}$ & $1.05 \pm 0.07$ \\
\hline \multicolumn{5}{|c|}{ Pazopanib } \\
\hline 1 & $1.05 \pm 0.15$ & $0.43 \pm 0.06^{*}$ & $1.01 \pm 0.11$ & $0.86 \pm 0.09$ \\
\hline \multicolumn{5}{|c|}{ Dabrafenib } \\
\hline 100 & $2.49 \pm 0.32 *$ & $0.41 \pm 0.05^{*}$ & $1.03 \pm 0.09$ & $0.28 \pm 0.01 *$ \\
\hline 50 & $2.42 \pm 0.21 *$ & $0.56 \pm 0.05^{*}$ & $1.69 \pm 0.30^{*}$ & $0.44 \pm 0.10^{*}$ \\
\hline 30 & $2.22 \pm 0.24 *$ & $0.71 \pm 0.03^{*}$ & $1.52 \pm 0.12 *$ & $0.59 \pm 0.07^{*}$ \\
\hline 20 & $1.59 \pm 0.05^{*}$ & $0.90 \pm 0.05$ & $1.31 \pm 0.02 *$ & $0.65 \pm 0.01 *$ \\
\hline 10 & $1.35 \pm 0.19^{*}$ & $0.94 \pm 0.04$ & $1.38 \pm 0.13^{*}$ & $0.97 \pm 0.07$ \\
\hline \multicolumn{5}{|c|}{ Cabozantinib } \\
\hline 30 & $1.55 \pm 0.06^{*}$ & $0.94 \pm 0.05$ & $1.35 \pm 0.18^{*}$ & $0.95 \pm 0.05$ \\
\hline 20 & $1.30 \pm 0.15^{*}$ & $0.92 \pm 0.05$ & $1.24 \pm 0.05^{*}$ & $0.94 \pm 0.06$ \\
\hline 10 & $1.46 \pm 0.08^{*}$ & $1.01 \pm 0.03$ & $1.23 \pm 0.11^{*}$ & $1.03 \pm 0.01$ \\
\hline \multicolumn{5}{|c|}{ Ruxolitinib } \\
\hline 100 & $0.71 \pm 0.08 *$ & $0.28 \pm 0.06^{*}$ & $1.17 \pm 0.12$ & $0.99 \pm 0.03$ \\
\hline 50 & $0.97 \pm 0.20$ & $0.49 \pm 0.06^{*}$ & $1.07 \pm 0.07$ & $0.87 \pm 0.08$ \\
\hline 30 & $1.00 \pm 0.09$ & $0.54 \pm 0.02 *$ & $1.08 \pm 0.09$ & $1.05 \pm 0.11$ \\
\hline 20 & $0.81 \pm 0.05$ & $0.62 \pm 0.05^{*}$ & $1.00 \pm 0.14$ & $0.90 \pm 0.13$ \\
\hline \multicolumn{5}{|c|}{ Ceritinib } \\
\hline 100 & $0.50 \pm 0.06^{*}$ & $0.08 \pm 0.02 *$ & $0.17 \pm 0.10^{*}$ & $0.03 \pm 0.02 *$ \\
\hline 50 & $1.21 \pm 0.12 *$ & $0.55 \pm 0.03^{*}$ & $0.77 \pm 0.07 *$ & $0.45 \pm 0.08^{*}$ \\
\hline 30 & $1.12 \pm 0.13$ & $0.88 \pm 0.05$ & $0.70 \pm 0.12^{*}$ & $0.41 \pm 0.18^{*}$ \\
\hline \multicolumn{5}{|c|}{ Imatinib } \\
\hline 100 & $0.90 \pm 0.12$ & $0.70 \pm 0.11^{*}$ & $1.04 \pm 0.06$ & $0.93 \pm 0.07$ \\
\hline 50 & $0.90 \pm 0.06$ & $0.67 \pm 0.03^{*}$ & $0.99 \pm 0.06$ & $0.90 \pm 0.01$ \\
\hline 30 & $0.93 \pm 0.04$ & $0.71 \pm 0.02 *$ & $0.98 \pm 0.02$ & $0.94 \pm 0.04$ \\
\hline \multicolumn{5}{|c|}{ Lenvatinib } \\
\hline 100 & $1.06 \pm 0.06$ & $0.74 \pm 0.04 *$ & $1.07 \pm 0.03$ & $0.99 \pm 0.03$ \\
\hline 50 & $0.93 \pm 0.01$ & $0.70 \pm 0.02 *$ & $0.96 \pm 0.03$ & $0.90 \pm 0.01$ \\
\hline 30 & $0.95 \pm 0.06$ & $0.76 \pm 0.05^{*}$ & $0.94 \pm 0.01$ & $0.86 \pm 0.04$ \\
\hline \multicolumn{5}{|c|}{ Idelalisib } \\
\hline 100 & $1.13 \pm 0.05$ & $0.66 \pm 0.03 *$ & $1.05 \pm 0.01$ & $0.65 \pm 0.03 *$ \\
\hline 50 & $1.00 \pm 0.08$ & $0.76 \pm 0.04 *$ & $0.97 \pm 0.04$ & $0.83 \pm 0.06$ \\
\hline \multicolumn{5}{|c|}{ Crizotinib } \\
\hline 100 & $0.97 \pm 0.14$ & $0.86 \pm 0.15$ & $1.09 \pm 0.12$ & $0.80 \pm 0.08^{*}$ \\
\hline
\end{tabular}

The signal from DMSO-treated samples was set as 1 . * $p<0.05$ as compared to DMSO-treated samples. The next lower concentrations showed no effects. KIs not listed also showed no effects

\section{KI effects on mitochondrial oxygen consumption}

Before drug treatment, the purity of mitochondria from selective rats was examined using Western blot. As shown in Supplementary Fig. 2A, the cytosolic protein GAPDH was only detectable in cytosolic fractions but not mitochondrial preparations. In contrast, the four mitochondrionspecific proteins including ATP5A, UQCRC2, SDHB and NDUFB8 were only detectable in mitochondrial preparations but not cytosolic fractions (Supplementary Fig. 2B). These results indicate that our mitochondria were devoid of cytosolic contamination.

Oxygen consumption is one of the most important functions of mitochondria that can be affected by hepatotoxic drugs. Figure 1 shows the mitochondrial oxygen consumption rate after KI treatment at the highest tested concentrations, and the dose dependent effects of 11 KIs that showed significant effects in Fig. 1 are presented in Table 3. The remaining data are included in Supplementary Table 1. Three KIs, including sorafenib, pazopanib and regorafenib, significantly affected oxygen consumption starting at concentrations equal to Cmax, while two KIs, dabrafenib and cabozantinib, began to show detrimental effects staring from tenfold Cmax (Table 3). Ruxolitinib and three other KIs (imatinib, ceritinib and lenvatinib) disrupted oxygen consumption starting from 20- and 30-fold Cmax, respectively. Idelalisib was non-effective until 50-fold Cmax, and crizotinib only affected oxygen consumption at 100-fold Cmax (Table 3).

At the highest tested concentrations, four drugs, including sorafenib, regorafenib, cabozantinib and dabrafenib caused significant increases of state 4 respiration (Fig. 1), indicating these drugs uncoupled oxidative phosphorylation (OXPHOS). Seven KIs decreased state 3 respiration driven by glutamate/malate (Fig. 1a), and four KIs suppressed state 3 respiration driven by succinate (Fig. 1b), indicating these drugs were likely inhibitors of OXPHOS. Of note, ceritinib caused almost $100 \%$ inhibition of mitochondrial oxygen consumption, albeit at 100-fold Cmax.

\section{KI effects on activities of RCC I-V}

The inhibitory effects of KIs on mitochondrial oxygen consumption might be due to the direct inhibition of RCC activities by KIs. To identify the specific RCCs inhibited by KIs, the activities of RCC I-V were measured using submitochondrial particles. KIs concentrations tested were the same as described for intact mitochondria. The results of all $31 \mathrm{KIs}$ at the highest tested concentrations are shown in Fig. 2a-e, and the dose dependent responses for the $11 \mathrm{KIs}$ that significantly inhibited RCCs were presented in Table 4. The "negative" results are included in Supplementary 

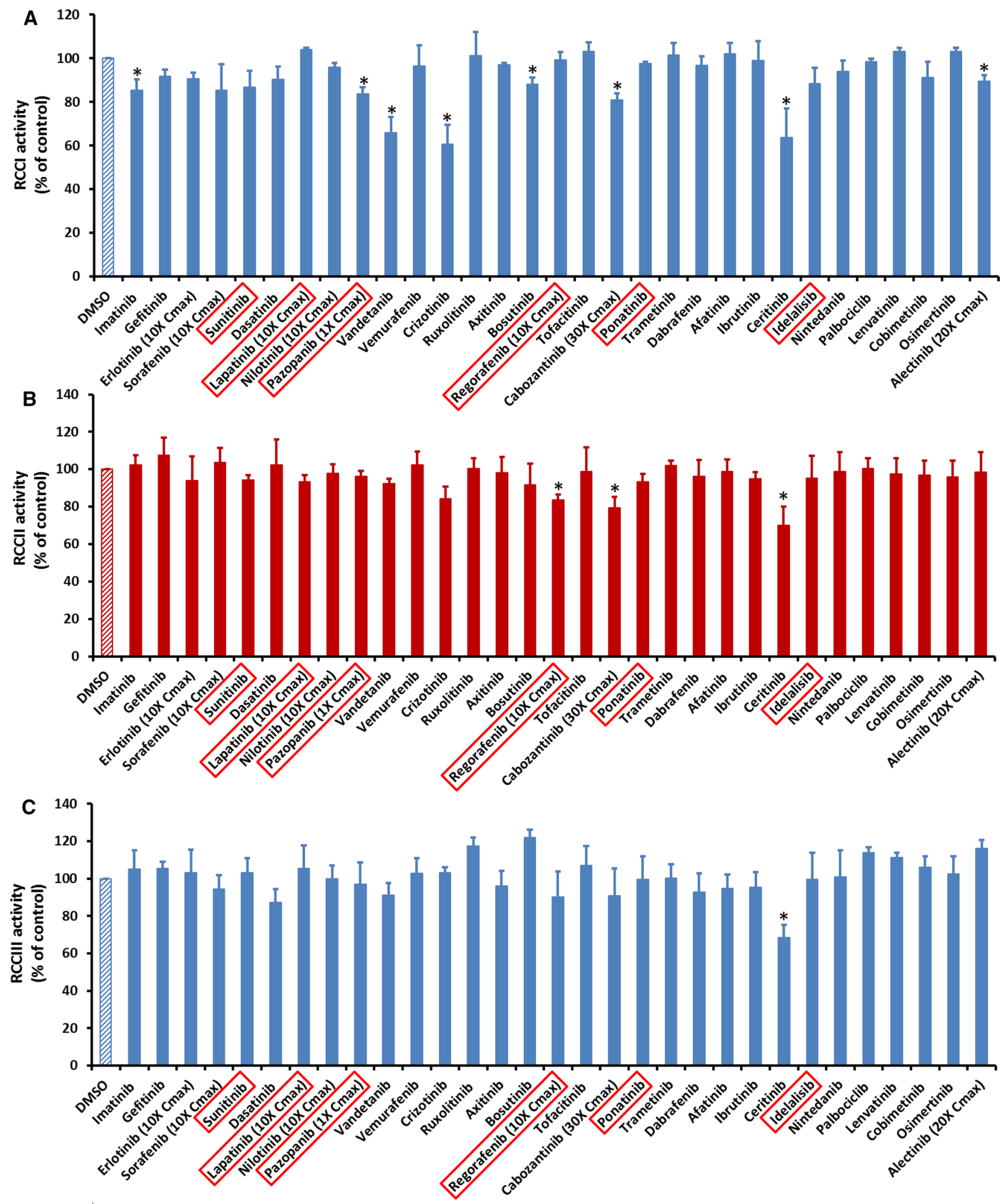

Fig. 2 KI effects on activities of RCC I-V. Submitochondrial particles were incubated with KIs at 100 -fold Cmax or the highest testable concentrations as. After $15 \mathrm{~min}$, the activities of RCC I-V were measured. a, b, c, d, e Represents the activity of RCC I, II, III, IV, and $\mathrm{V}$, respectively. The drugs in the $X$-axis were listed according to their approval date. The six KIs with a black box warning for hepato- toxicity were highlighted in a red box. The $Y$-axis represents the percentage of RCC activity after KI treatment as compared to DMSOtreated samples which were set as 100. Data are means and standard derivations from three independent experiments. $* p<0.05$ as compared to DMSO-treated samples (color figure online) 

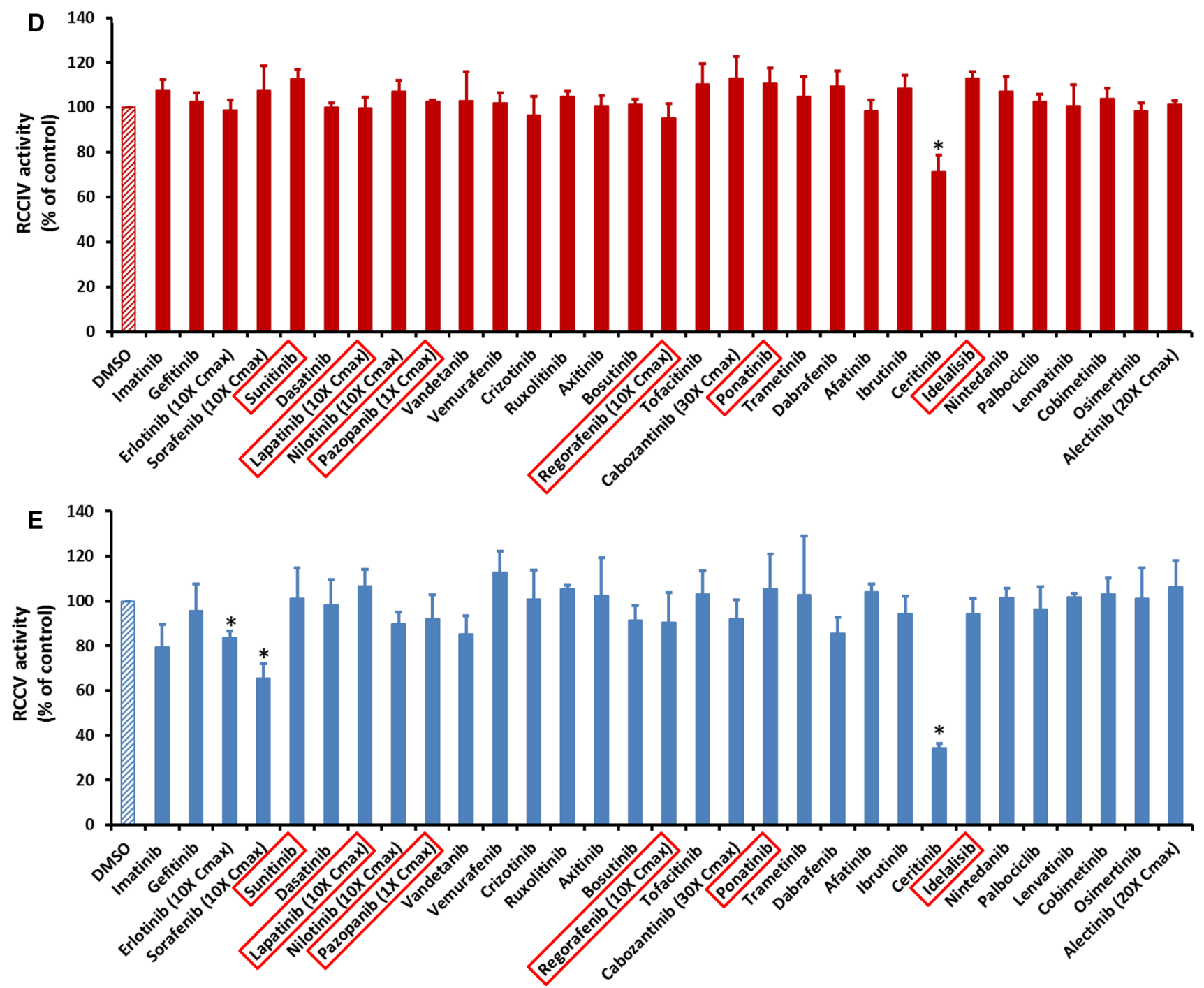

Fig. 2 continued

Table 1. Pazopanib was the only KI that inhibited RCCs at concentrations equal to Cmax, and sorafenib was the next toxic KIs as it started to inhibit RCCs at fivefold Cmax. Regorafenib and erlotinib began to suppress RCCs at 10fold Cmax, and the other KIs showed detrimental effect only at concentrations equal or larger than 20 -fold Cmax. At the highest tested concentrations, the number of KIs inhibiting RCC I, II, III, IV, and V was 8, 3, 1, 1, and 3, respectively. It was evident that RCC I was the major target for KI inhibition. The drug ceritinib was the only KI that caused inhibition of all five RCCs, and cabozantinib inhibited both RCC I and RCC II. For other KIs with RCC inhibition, the effects appeared protein complex-specific. For example, imatinib, regorafenib, and sorafenib only inhibited RCC I, RCC II and RCC V, respectively, but not other RCCs.

\section{KI effects on MMP}

Uncoupling of mitochondrial respiration can disrupt MMP. Among 31 KIs tested, only five drugs including regorafenib, ceritinib, dabrafenib, sorafenib and cabozantinib caused a decrease in MMP at the highest concentrations tested (Fig. 3a), and the remaining drugs showed no effects at any of the concentrations tested (Fig. 3b; Supplementary Table 1). Among the five effective drugs, ceritinib showed the strongest potential. Dose-response studies showed that sorafenib and regorafenib started to decrease MMP at onefold Cmax, and cabozantinib and dabrafenib at tenfold and 20-fold Cmax, respectively, while ceritinib showed no effects at concentrations lower than 100-fold Cmax (Fig. 3c). 
Table 4 Dose response of KI effects on activities of RCC I-V

\begin{tabular}{|c|c|c|c|c|c|}
\hline \multirow[t]{2}{*}{ Drugs (fold of Cmax) } & \multicolumn{5}{|c|}{ RCC activity ( $100 \%$ of control) } \\
\hline & I & II & III & IV & V \\
\hline \multicolumn{6}{|l|}{ Pazopanib } \\
\hline 1 & $83.77 \pm 2.98^{*}$ & $96.12 \pm 2.96$ & $96.90 \pm 11.69$ & $102.68 \pm 0.57$ & $92.22 \pm 10.47$ \\
\hline \multicolumn{6}{|l|}{ Sorafenib } \\
\hline 10 & $85.45 \pm 11.93$ & $103.68 \pm 7.88$ & $94.31 \pm 7.47$ & $107.65 \pm 10.77$ & $65.55 \pm 6.34 *$ \\
\hline 5 & $87.23 \pm 10.61$ & $101.40 \pm 6.29$ & $97.60 \pm 5.50$ & $101.55 \pm 3.61$ & $78.30 \pm 4.68 *$ \\
\hline \multicolumn{6}{|l|}{ Erlotinib } \\
\hline 10 & $90.62 \pm 2.87$ & $93.97 \pm 12.92$ & $103.11 \pm 12.25$ & $98.77 \pm 4.53$ & $83.52 \pm 2.96^{*}$ \\
\hline \multicolumn{6}{|l|}{ Regorafenib } \\
\hline 10 & $99.37 \pm 3.35$ & $83.50 \pm 2.91 *$ & $90.31 \pm 13.61$ & $95.37 \pm 6.45$ & $90.36 \pm 13.39$ \\
\hline \multicolumn{6}{|l|}{ Crizotinib } \\
\hline 100 & $60.51 \pm 8.90^{*}$ & $84.10 \pm 6.53$ & $103.28 \pm 2.85$ & $96.60 \pm 8.40$ & $100.80 \pm 13.09$ \\
\hline 50 & $71.46 \pm 4.28^{*}$ & $90.31 \pm 4.38$ & $98.02 \pm 7.16$ & $103.77 \pm 5.67$ & $91.95 \pm 5.63$ \\
\hline 30 & $76.48 \pm 2.18^{*}$ & $95.03 \pm 1.23$ & $106.26 \pm 5.79$ & $96.65 \pm 4.14$ & $98.65 \pm 2.98$ \\
\hline 20 & $86.52 \pm 2.70 *$ & $100.85 \pm 3.65$ & $99.67 \pm 8.65$ & $94.03 \pm 9.67$ & $95.77 \pm 7.64$ \\
\hline \multicolumn{6}{|l|}{ Alectinib } \\
\hline 20 & $89.39 \pm 2.98^{*}$ & $98.44 \pm 10.74$ & $116.05 \pm 4.72$ & $101.46 \pm 1.66$ & $106.30 \pm 11.74$ \\
\hline \multicolumn{6}{|l|}{ Cabozantinib } \\
\hline 30 & $80.97 \pm 2.99 *$ & $79.26 \pm 6.01 *$ & $90.73 \pm 14.60$ & $113.10 \pm 9.48$ & $91.99 \pm 8.51$ \\
\hline \multicolumn{6}{|l|}{ Ceritinib } \\
\hline 100 & $63.74 \pm 13.12 *$ & $69.91 \pm 10.00 *$ & $68.48 \pm 6.59 *$ & $71.46 \pm 7.39 *$ & $34.25 \pm 2.09 *$ \\
\hline 50 & $94.04 \pm 3.32$ & $89.14 \pm 6.26$ & $103.80 \pm 5.19$ & $82.33 \pm 3.68^{*}$ & $71.23 \pm 9.65^{*}$ \\
\hline \multicolumn{6}{|l|}{ Vandetanib } \\
\hline 100 & $65.88 \pm 7.15^{*}$ & $92.39 \pm 2.47$ & $91.23 \pm 6.54$ & $103.06 \pm 12.96$ & $85.15 \pm 8.36$ \\
\hline 50 & $87.71 \pm 0.58 *$ & $90.03 \pm 5.23$ & $99.20 \pm 5.61$ & $100.56 \pm 2.67$ & $95.65 \pm 4.66$ \\
\hline \multicolumn{6}{|l|}{ Imatinib } \\
\hline 100 & $85.30 \pm 5.13^{*}$ & $102.36 \pm 5.33$ & $104.96 \pm 10.18$ & $107.65 \pm 4.65$ & $79.34 \pm 10.06$ \\
\hline \multicolumn{6}{|l|}{ Bosutinib } \\
\hline 100 & $88.03 \pm 3.01 *$ & $91.74 \pm 11.35$ & $101.81 \pm 4.43$ & $101.25 \pm 2.26$ & $91.53 \pm 6.49$ \\
\hline
\end{tabular}

The signal from DMSO-treated samples was set as $100 . * p<0.05$ as compared to DMSO-treated samples. The next lower concentrations showed no effects. KIs not listed also showed no effects

\section{KI effects on mitochondrial swelling}

Altered permeability of the mitochondrial inner membrane can lead to swelling. Figure 4 a shows that seven KIs including regorafenib, sorafenib, dabrafenib, cabozantinib, ceritinib, crizotinib and vandetanib caused significant mitochondrial swelling at the highest concentrations tested. Other drugs tested showed no effects on mitochondrial swelling at any of the concentrations tested (Fig. 4b; Supplementary Table 1). The dose response of five effective KIs is presented in Fig. 4c. Only one drug regorafenib caused mitochondrial swelling at onefold Cmax, and sorafenib caused swelling at 2.5-fold Cmax (Supplementary Table 1). Of note, though regorafenib at onefold Cmax caused almost maximal swelling, it was not possible for us to test it reliably at the next higher concentrations (2.5-fold Cmax), because absorbance at $535 \mathrm{~nm}$ of the drug itself was observed. This was also the case for sorafenib. Therefore, the dose response curves were not presented in the main text. Figure $4 \mathrm{c}$ shows that dabrafenib and cabozantinib started to cause swelling at tenfold and 20-fold Cmax, respectively. The remaining three drugs including ceritinib, crizotinib, and vandetanib only caused swelling at 100 -fold Cmax.

\section{KI effects on cytochrome c release}

Changes in the permeability of mitochondrial outer membrane can lead to cytochrome c release. Figure 5a shows that $11 \mathrm{KIs}$ induced cytochrome $\mathrm{c}$ release at the highest concentrations tested, and the remaining $20 \mathrm{KIs}$ were ineffective. The dose response of seven KIs was shown in Fig. 5b-d. Only regorafenib started to trigger cytochrome c 
release at onefold Cmax (Fig. 5b), and two drugs, sorafenib and dabrafenib, caused cytochrome c release starting at fivefold Cmax (Fig. 5c, d). Lapatinib caused cytochrome release only at tenfold Cmax, though higher concentrations cannot be tested due to its limited solubility (Supplementary Table 1). Four KIs including ceritinib, ruxolitinib, crizotinib and imatinib began to cause cytochrome $\mathrm{c}$ at 50-fold Cmax (Fig. 5d), and three KIs including vandetanib, tofacitinib, osimertinib only showed significant effects at 100-fold Cmax. Among all 31 KIs tested, ceritinib caused the most significant cytochrome $\mathrm{c}$ release, that is, $33 \%$ of total cytochrome c was leaked after treatment, though this occurred at 100-fold Cmax (Fig. 5d).

\section{KI effects on ROS production in mitochondria}

ROS is produced at low levels during normal mitochondrial respiration. Drug-induced inhibition of oxygen consumption can lead to ROS overproduction. As shown in Fig. 6a, calcium chloride induced a threefold increase in ROS production. However, none of the KIs caused ROS over production in mitochondria at 1 to 10 fold Cmax (Supplementary Table 1). At the highest tested concentrations, four KIs including crizotinib, dabrafenib, ceritinib, and osimertinib caused significantly ROS production (Fig. 6a). The dose response of these four KIs is shown in Fig. 6b. While crizotinib and osimertinib trigger ROS overproduction beginning from 20-fold and 30-fold Cmax, respectively, dabrafenib and ceritinib only started to show significant effects from 50-fold Cmax. The magnitude of ROS production induced by osimertinib at 100-fold Cmax was even slightly higher than those caused by calcium chloride.

\section{Prediction of KI hepatotoxicity using mitotoxicity as compared to daily dose and Cmax}

Table 5 shows the results of using KI mitotoxicity to predict hepatotoxicity. For this purpose, the drugs that cannot be tested at higher concentrations due to solubility issues will be either considered as mitotoxicity positive if they showed positive mitotoxicity at lower concentrations or excluded at untestable concentrations when low concentrations showed no mitotoxicity. At 100-fold Cmax with intact mitochondria, the PPV was $67 \%$ and NPV was only $31 \%$, and the corresponding sensitivity and specificity was 53 and 44\%, respectively. At 100-fold Cmax with both intact mitochondria and submitochondrial particles, though the specificity was not affect, other parameters were all increased, indicating that inclusion of submitochondrial particles enhanced the predictive power. At onefold Cmax with intact mitochondria, the PPV and specificity was all $100 \%$, but NPV and sensitivity was only 32 and $13 \%$, respectively. The results at other concentrations were presented in Supplementary Table 2. The overall results were that though the PPV was decent, the NPV was always no greater than $50 \%$. Interestingly, using a cutoff of $\mathrm{Cmax} \geq 1.1 \mu \mathrm{M}$ (Shah et al. 2015) or daily dose $\geq 100 \mathrm{mg}$ (Weng et al. 2015b), similar predictive results were obtained as using mitotoxicity data (Supplementary Table 2).

\section{Discussion}

Though several KIs have been investigated in previous reports regarding mitochondrial toxicity, a comprehensive study involving all FDA approved KIs is not yet available. In addition, KI effects from previous reports cannot be compared directly, as different platforms were used. This study not only significantly expanded the number of KIs tested for mitochondrial toxicity, but also provided additional data on mitochondrial liabilities such as ROS production and RCC activities that were not examined in previous studies.

Some endpoints measured in this study can be related. For example, an uncoupler can disrupt inner membrane potential, and RCC inhibition may cause ROS overproduction, and cytochrome c release can affect oxygen consumption. However, the relation is not always causative. For example, the increase in ROS production caused by mild RCC inhibition can be antagonized by endogenous antioxidant. Another example is that RCC inhibition can decrease oxygen consumption and uncoupling can enhance oxygen consumption, and therefore if a KI is both a RCC inhibitor and an uncoupler, such as sorafenib, the net effects on oxygen consumption can be complicated. Further studies are needed to clarify the relations among mitochondrial changes caused by KIs.

Of the 31 KIs examined only three including sorafenib, regorafenib and pazopanib, all of which are DILI positive, caused significant mitochondrial toxicity at concentrations equal to the Cmax. At similar concentrations, sorafenib has been shown to be an uncoupler and inhibitor of OXPHOS in isolated rat heart mitochondria (Will et al. 2008) and regorafenib is reported to be a pure uncoupler without inhibition of OXPHOS in isolated rat liver mitochondria (Weng et al. 2015a), while no data are available for pazopanib. This study showed that sorafenib affected rat liver mitochondria in a similar manner as reported in rat heart mitochondria (Will et al. 2008). Sorafenib was also reported to be a strong mitochondrial toxicant in neuroblastoma cells (Bull et al. 2012). Collectively, these data suggest that sorafenib-induced effects on mitochondria are not tissue specific, and that mitochondrial toxicity may contribute to various types of organ toxicity associated with sorafenib. This study also identified an additional mitochondrial liability of regorafenib not reported in the previous study 

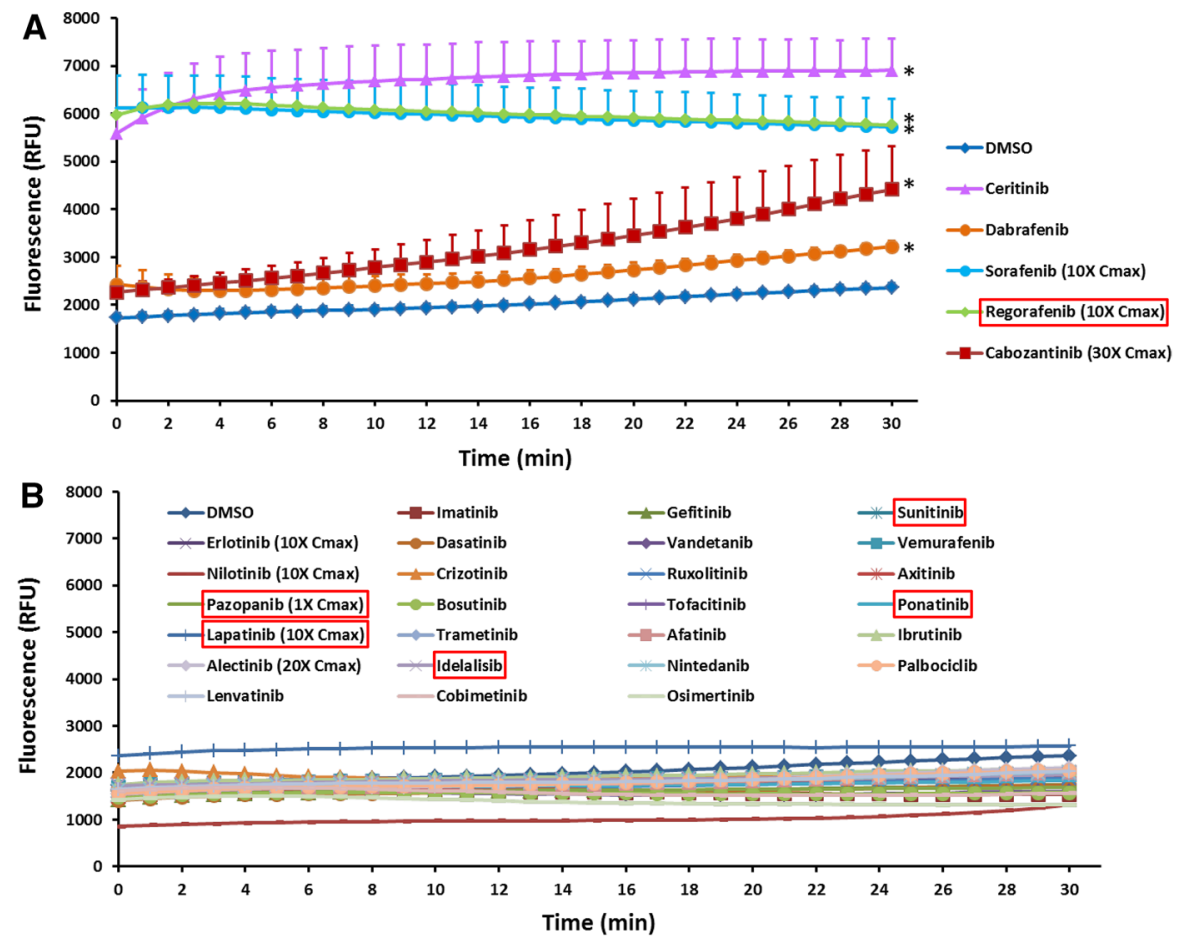

\section{Regorafenib}
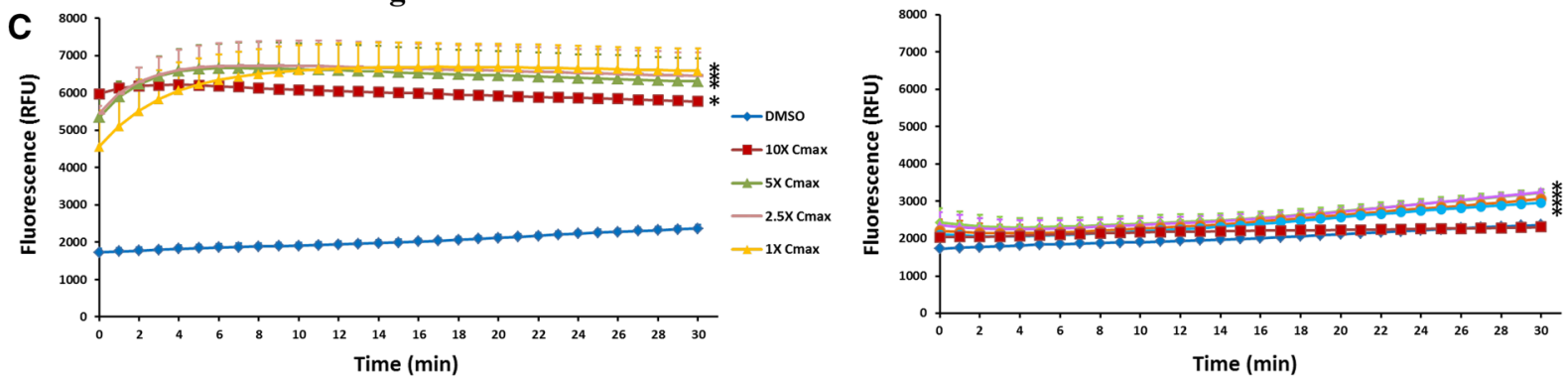

$\rightarrow$ DMso

$\rightarrow-100 x C_{\text {max }}$

$=50 x c_{\max }$

$-30 x$ cmax

Sorafenib
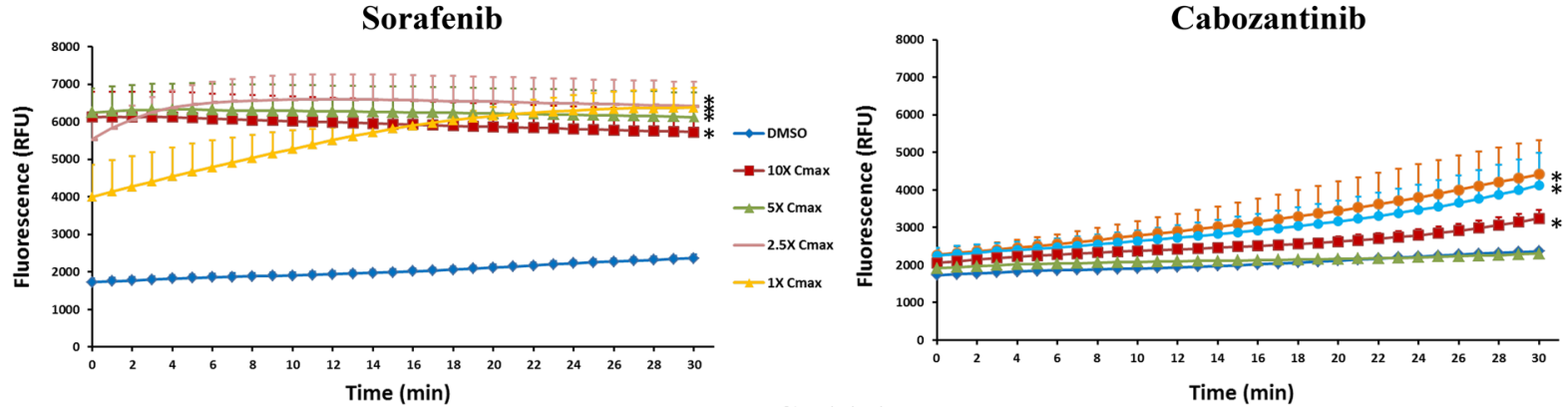

$\rightarrow$ DMsO

$\rightarrow 30 x \mathrm{Cmax}$

Ceritinib

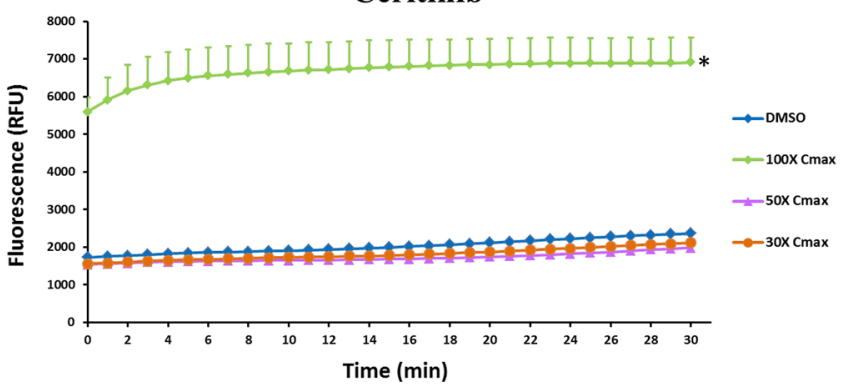


4Fig. 3 KI effects on mitochondrial inner membrane potential (MMP). Rat liver mitochondria were supplemented with $10 \mu \mathrm{M}$ Rhodamine 123 and incubated with KIs at 100 -fold Cmax or the highest testable concentrations as indicated. The fluorescence (excitation $485 \mathrm{~nm}$, emission $535 \mathrm{~nm}$ ) was recorded every 1 min for $30 \mathrm{~min}$ and presented in the $Y$-axis $(\mathbf{a}, \mathbf{b})$. c Shows the dose response of five KIs. In $\mathbf{a}, \mathbf{c}$, data are means and standard derivations from three separate experiments. $* p<0.05$ as compared to DMSO-treated samples at the end of experiments. In $\mathbf{b}$, data are means of two independent experiments

(Weng et al. 2015a), that is, regorafenib caused cytochrome c release at a concentration equal to Cmax. Of note is that the chemical structures of sorafenib and regorafenib are very similar (Supplementary Fig. 1). This study found that both drugs were uncouplers and both caused cytochrome $\mathrm{c}$ release, a decreased in MMP, and mitochondrial swelling, while neither of them affected ROS production. It appears likely that sorafenib and regorafenib share chemical properties that are associated with mitochondrial injury leading to organ toxicity.

The drug pazopanib, which carries a BBW for DILI on its labeling, has the highest $\mathrm{Cmax}(133 \mu \mathrm{M})$ among all KIs examined and it is not possible to test its effects at more than onefold Cmax concentrations due to the solubility issue. At onefold Cmax, pazopanib inhibited state 3 mitochondrial respiration driven by glutamate/malate by over $50 \%$, but showed no effects on succinate-driven respiration, indicating that pazopanib may specifically inhibit RCC I. Indeed, with submitochondrial particles, pazopanib was confirmed to be an inhibitor of RCC I but not other RCCs. These data suggest that selective inhibition of RCC I may contribute to the pathogenesis of pazopanib hepatotoxicity.

The drugs ponatinib and sunitinib have BBWs for DILI in the labeling. However, both drugs showed no effects on intact mitochondria or submitochondrial particles even at the highest concentrations tested, indicating that mitochondrial liability at 100-Cmax would not predict DILI positive KI with high confidence. Alternative mechanisms, such as reactive metabolites, may account for DILI associated with these two drugs. Our findings on sunitinib with intact mitochondria are consistent with previous reports carried out either in rat heart mitochondria (Will et al. 2008) or mouse liver mitochondria (Porceddu et al. 2012). However, in the latter mouse study, sunitinib was considered as mitochondrial toxicity positive, as the authors determined that the Cmax of sunitinib was $70.8 \mu \mathrm{M}$ (Porceddu et al. $2012)$, which is sharp contrast with ours $(0.12 \mu \mathrm{M})$ and others' $(0.25 \mu \mathrm{M})$ (Will et al. 2008). If the true Cmax of sunitinib was $70.8 \mu \mathrm{M}$, it would not be feasible for us to have it tested at 100 -fold $\mathrm{Cmax}$ due to the solubility issue.

The DILI negative drug vandetanib inhibited RCC I starting at 50-fold Cmax, but had no effects on mitochondrial oxygen consumption even at 100-fold Cmax. The likely reason for this discrepancy is that RCC activities need to be measured using submitochondrial particles, while oxygen consumption is determined using intact mitochondria. Differential effects of chemicals on whole mitochondria and submitochondrial particles have been reported previously, and one likely reason, among many others, was that the compound may not easily cross the inner membrane and therefore RCC proteins were protected from the drug's effects with intact mitochondria (Weng et al. 2014). Further investigations are needed to confirm if this is true for vandetanib and other KIs showing similar effects. On the other hand, another DILI negative drug dabrafenib caused significant inhibition of state 3 respiration in intact mitochondria but showed no effects on RCC activities in submitochondrial particles. The likely reason for this discrepancy is that dabrafenib also caused cytochrome c release leading to suppressed oxygen consumption. It should be pointed out that these two DILI negative drugs indeed caused various types of mitochondrial injury at 100 -fold Cmax, implying that mitochondrial liability at $100-\mathrm{Cmax}$ is not a good predictor of DILI negative KIs. Further supporting this premise, the DILI negative drugs dabrafenib, cabozantinib, ruxolitinib, osimertinib, and ibrutinib also showed significant toxicity to intact mitochondria at concentrations starting from 5-fold, 10-fold, 20-fold, 30-fold, and 50-fold Cmax, respectively. Interestingly, one of the veterinary KIs oclacitinib has not been associated with DILI in animals. However, at 50-fold Cmax, oclacitinib caused significant cytochrome $\mathrm{c}$ release from intact mitochondria (data not shown). Taken together, these data indicate that mitochondrial toxicity was too sensitive in predicting DILI negative KIs. However, the possibility that the DILI potential of these KIs has not been fully recognized from clinical use cannot be ruled out.

The predictive power of the mitochondrial model for KI hepatotoxicity presented here is in contrast to a previous report showing that human DILI in general can be predicted with high confidence using in vitro mitochondrial endpoints measured at 100-fold Cmax (Porceddu et al. 2012). The likely reason for this discrepancy is that $\mathrm{KI}$ hepatotoxicity may differ from DILI caused by other therapeutic groups of drugs. To our knowledge, though several in vitro systems have been proposed to be effective in predicting a compound's DILI potential, no previous studies have included all FDA approved KIs for this purpose. Our findings highlight the challenge posed by KIs for predicting DILI using mitochondrial assays and warrant further investigations using alternative in vitro systems.

An interesting observation is that mitochondrial toxicity at onefold and 2.5-fold Cmax showed 100\% PPV and $100 \%$ specificity in predicting KI hepatotoxicity, though the NPV and sensitivity were 32 and 14\%, respectively. 

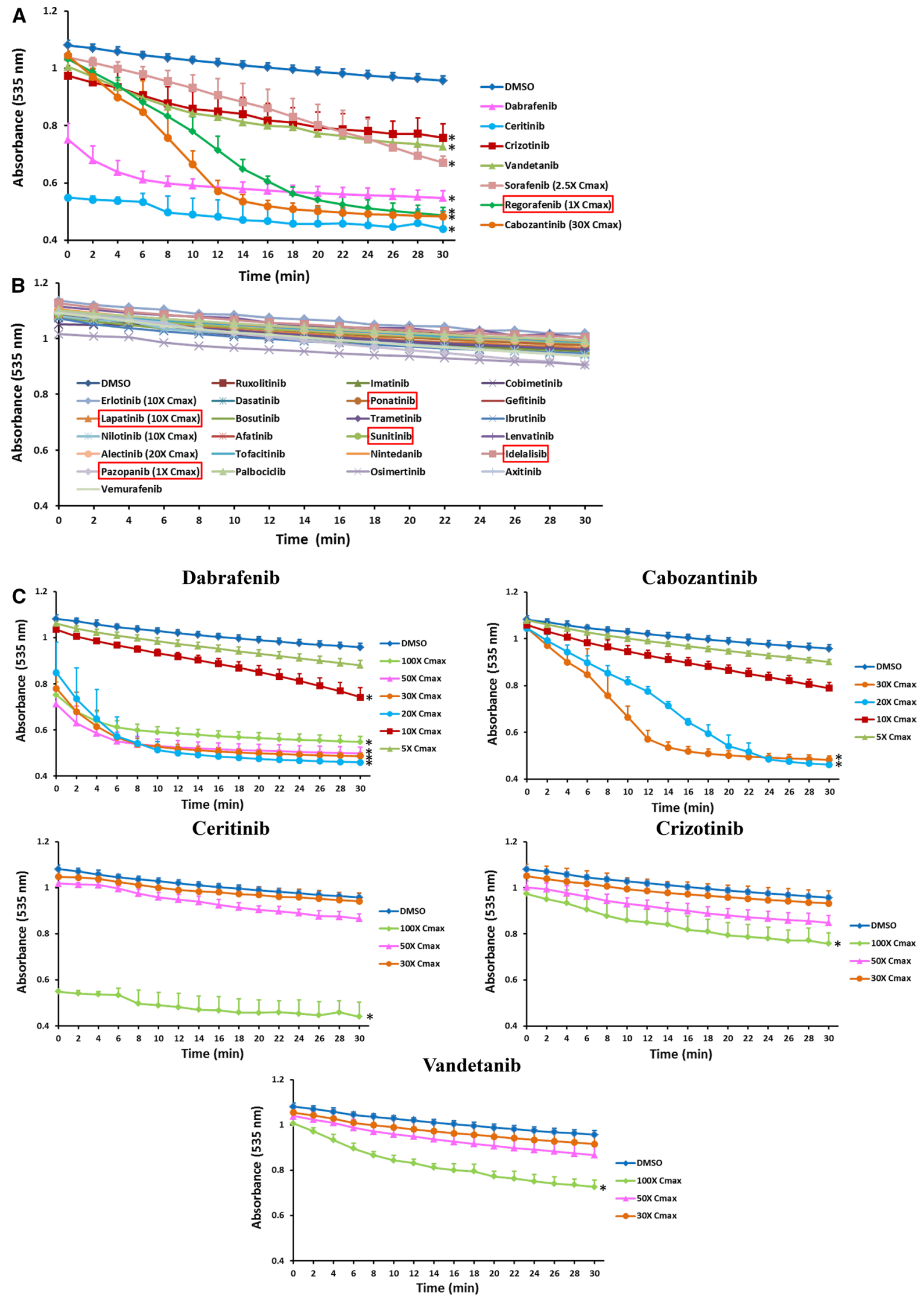
\Fig. 4 KI effects on mitochondrial swelling. Rat liver mitochondria were supplemented with $25 \mu \mathrm{M}$ calcium chloride and incubated with KIs at 100 -fold Cmax or the highest testable concentrations as indicated. The six KIs with a black box warning for hepatotoxicity were highlighted in a red box. Mitochondrial swelling was determined by monitoring the decrease in absorbance at $535 \mathrm{~nm}$ every 2 min for 30 min. c Shows the dose response of five KIs. a, c Data are means and standard derivations of three separate experiments. $* p<0.05$ as compared to DMSO-treated samples at the end of experiments. b Data are means of two independent experiments (color figure online)

This indicates that if a drug causes mitotoxicity in any of the endpoints assessed at concentrations equal to Cmax, it will very likely be DILI positive; however, a drug being non-mitotoxic at Cmax does not mean that it will be DILI negative, as alternative or indirect mechanisms may be involved. It appears that mitotoxicity at 1-2.5-fold Cmax can be used to help identify hepatotoxic KIs as an early safety screen during drug discovery.

Many drugs impair mitochondrial functions contributing to DILI pathogenesis, although only a few have been directly tested in vivo or in humans (McGill et al. 2012). Drugs affecting mitochondrial functions in vitro at clinically relevant concentrations are expected to have a high likelihood of doing so under in vivo conditions. This study identified several KIs causing significant mitochondrial injury at concentrations equal to $\mathrm{Cmax}$ or only at

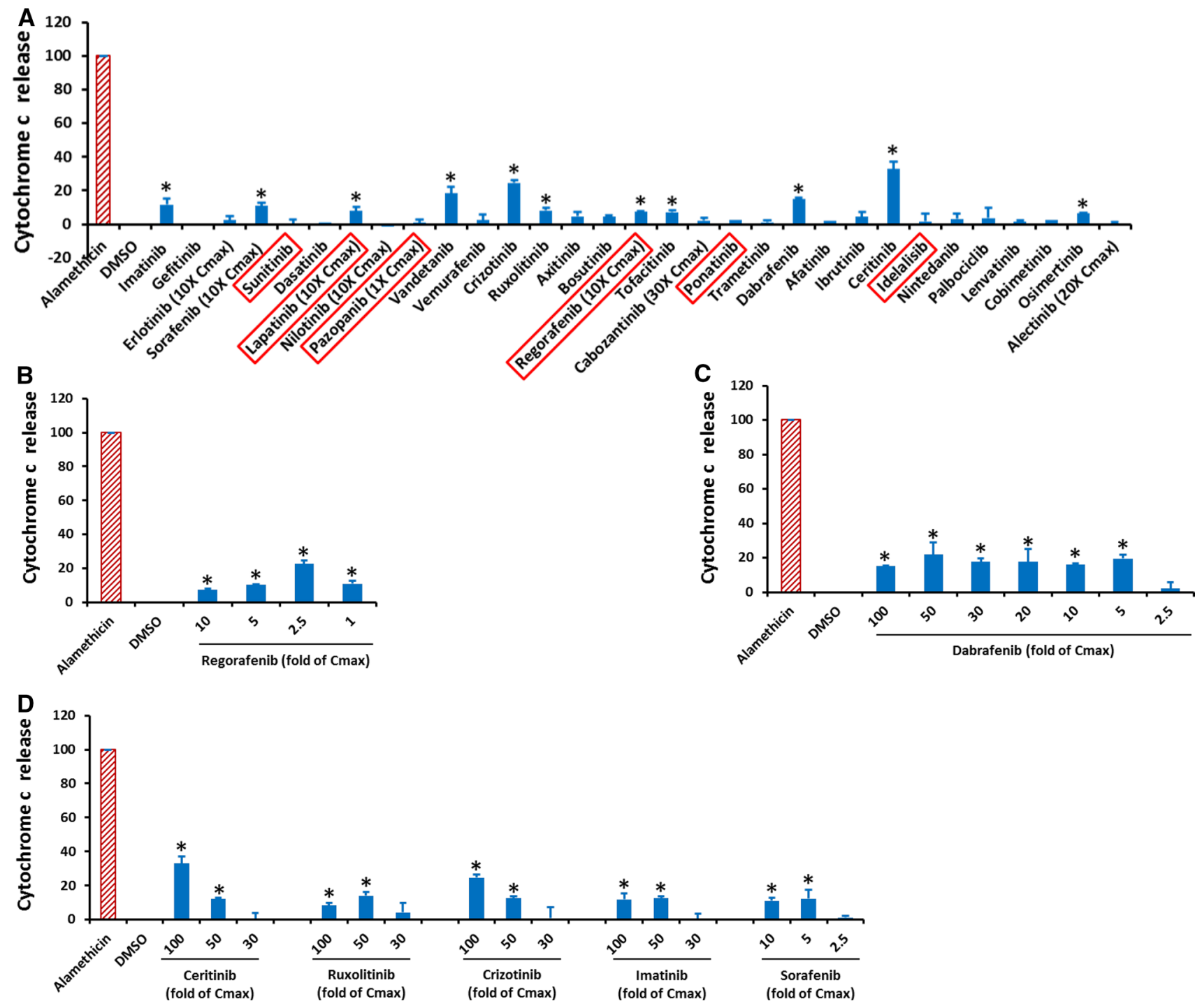

Fig. 5 KI effects on cytochrome c release. Rat liver mitochondria were incubated with KIs at 100 -fold Cmax or the highest testable concentrations as indicated. Alamethicin was used to induce complete cytochrome c release, and its signal was set as 100. After $30 \mathrm{~min}$, the mitochondria were pelleted and cytochrome $\mathrm{c}$ level in the supernatant was determined using an ELISA Kit. The drugs in the $X$-axis were listed according to their approval date. The six KIs with a black box warning for hepatotoxicity were highlighted in a red box (a). b-d Are the dose response of seven KIs. Data are means and standard derivations from three independent experiments. ${ }^{*} p<0.05$ as compared to DMSO-treated samples (color figure online) 

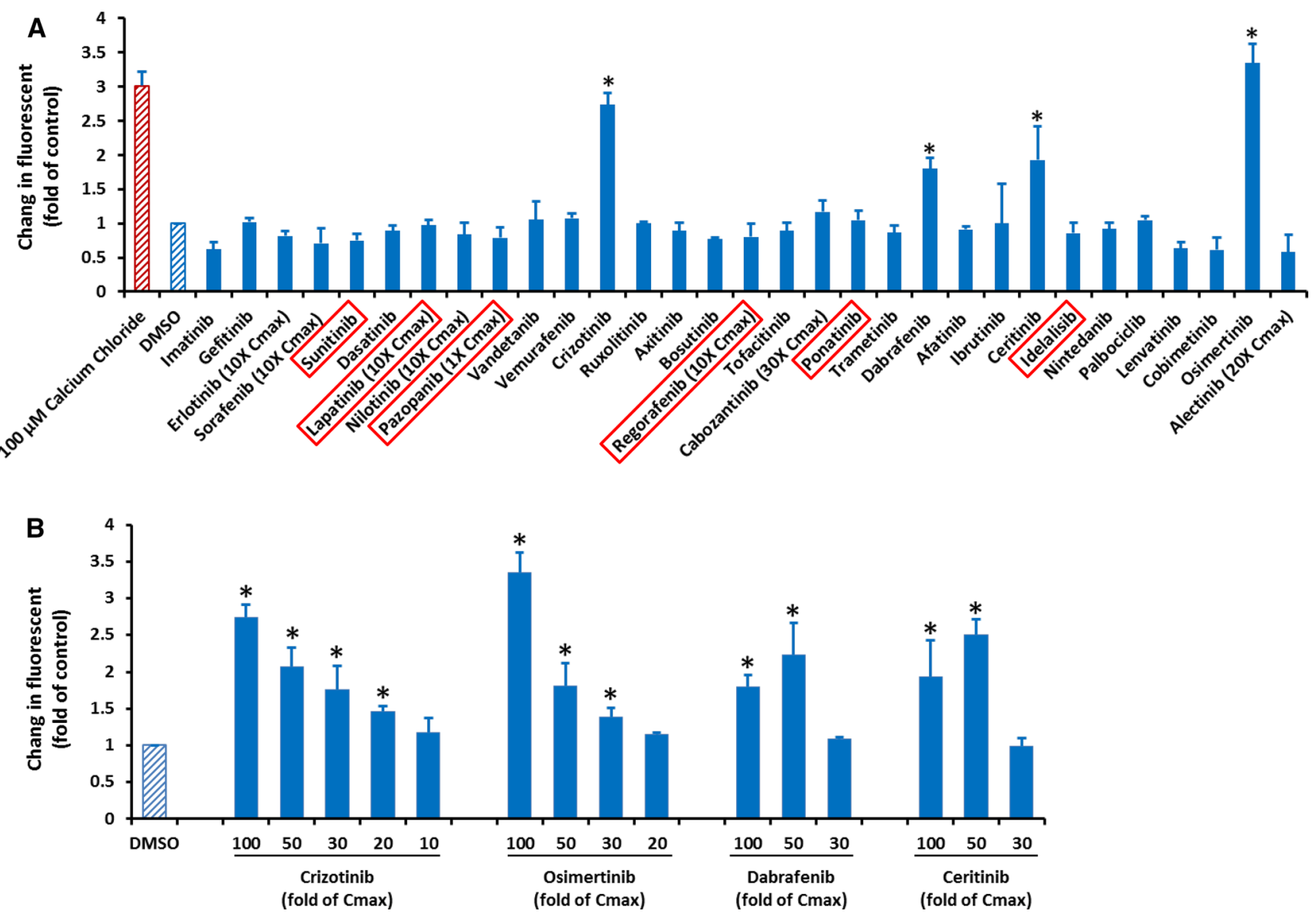

Fig. 6 KI effects on ROS production in mitochondria. Rat liver mitochondria were loaded with $4 \mu \mathrm{M}$ CM-H2DCFDA and then treated with KIs at 100-fold Cmax or the highest testable concentrations as indicated. Calcium chloride was used as a positive control. The fluorescence (excitation $490 \mathrm{~nm}$, emission $530 \mathrm{~nm}$ ) was measured every $1 \mathrm{~min}$ for $30 \mathrm{~min}$. The signal from DMSO-treated samples was set as 1 . The drugs in the $X$-axis were listed according to their approval date. The six KIs with a black box warning for hepatotoxicity were highlighted in a red box (a). b Shows the dose response of four KIs. Data are means and standard derivations from three independent experiments. ${ }^{*} p<0.05$ as compared to DMSO-treated samples (color figure online)

Table 5 Prediction of KI hepatotoxicity by using mitotoxicity at different concentrations

\begin{tabular}{|c|c|c|c|c|c|c|c|c|}
\hline Fold of Cmax & Test system & Mitotoxicity & DILI+ & DILI- & PPV & NPV & Sensitivity & Specificity \\
\hline \multirow[t]{4}{*}{100} & \multirow[t]{2}{*}{ Intact-mito (excluded 3 drugs) } & + & 10 & 5 & \multirow[t]{2}{*}{$67 \%$} & \multirow[t]{2}{*}{$31 \%$} & \multirow[t]{2}{*}{$53 \%$} & \multirow[t]{2}{*}{$44 \%$} \\
\hline & & - & 9 & 4 & & & & \\
\hline & \multirow[t]{2}{*}{ Intact and Sub-mito (excluded 1 drug) } & + & 13 & 5 & \multirow[t]{2}{*}{$72 \%$} & \multirow[t]{2}{*}{$33 \%$} & \multirow[t]{2}{*}{$62 \%$} & \multirow[t]{2}{*}{$44 \%$} \\
\hline & & - & 8 & 4 & & & & \\
\hline \multirow[t]{4}{*}{1} & \multirow[t]{2}{*}{ Intact-mito } & + & 3 & 0 & \multirow[t]{2}{*}{$100 \%$} & \multirow[t]{2}{*}{$32 \%$} & \multirow[t]{2}{*}{$14 \%$} & \multirow[t]{2}{*}{$100 \%$} \\
\hline & & - & 19 & 9 & & & & \\
\hline & \multirow[t]{2}{*}{ Intact and Sub-mito } & + & 3 & 0 & \multirow[t]{2}{*}{$100 \%$} & \multirow[t]{2}{*}{$32 \%$} & \multirow[t]{2}{*}{$14 \%$} & \multirow[t]{2}{*}{$100 \%$} \\
\hline & & - & 19 & 9 & & & & \\
\hline
\end{tabular}

$P P V$ positive predictive value, $N P V$ negative predictive value, $D I L I$ drug-induced liver injury. A drug is considered as mitotoxicity positive $(+)$ if it causes at least one type of mitochondrial injury. Intact-mito: data from whole mitochondria; Sub-mito: data from submitochondrial particles

several fold of Cmax. These drugs can serve as good candidates for future exploratory in vivo studies. Mitochondrion-enriched molecules, such as mitochondrial DNA and cytochrome c, can be released into the blood when liver injury is induced by drugs causing mitochondrial damage, and circulating mitochondrial molecules have 
been shown to be novel biomarkers for DILI induced by mitotoxic drugs (Miller et al. 2008; Shi et al. 2015). It is worthwhile to examine if KI hepatotoxicity can be monitored using circulating mitochondrial biomarkers. The KIs that showed the strongest mitochondrial toxicities, such as sorafenib, regorafenib, and pazopanib, are particularly suitable for testing this hypothesis in future investigations.

It should be pointed out that the DILI potential of some drugs was not fully recognized until being marketed for many years (Rivkees 2010). As most KIs were approved very recently, the DILI potential of these drugs may not be thoroughly understood until more time has passed. Therefore, the predictive results presented here may change over time as more patients take these drugs. Nevertheless, our findings demonstrate that mitochondrial toxicity has limited predictive power for human DILI observed in clinical trials and post-marketing, studies that laid the foundation for drug approval and labeling. Alternative in vitro approaches, such as primary hepatocytes or human-induced pluripotent stem cell-derived hepatocytes, may partially overcome key disadvantages of isolated mitochondria, such as the lack of major drug metabolizing enzymes or regeneration/compensatory mechanisms. Given that human DILI remains poorly predicted by animal models, due possibly to the polymorphic nature of the human liver, further investigations into such in vitro approaches with addition of drug metabolizing enzymes or use of more human-based systems may likely prove worthwhile in future studies.

Acknowledgements This project was partially supported by the US FDA's Office of Women's Health. Drs. Jun Zhang and Matthew White are supported by the Research Participation Program at the National Center for Toxicological Research administrated by the Oak Ridge Institute for Science and Education through an interagency agreement between the U.S. Department of Energy and the U.S. FDA.

Open Access This article is distributed under the terms of the Creative Commons Attribution 4.0 International License (http://creativecommons.org/licenses/by/4.0/), which permits unrestricted use, distribution, and reproduction in any medium, provided you give appropriate credit to the original author(s) and the source, provide a link to the Creative Commons license, and indicate if changes were made.

\section{References}

Aleo MD, Luo Y, Swiss R, Bonin PD, Potter DM, Will Y (2014) Human drug-induced liver injury severity is highly associated with dual inhibition of liver mitochondrial function and bile salt export pump. Hepatology 60(3):1015-1022. doi:10.1002/hep.27206

Bull VH, Rajalingam K, Thiede B (2012) Sorafenib-induced mitochondrial complex I inactivation and cell death in human neuroblastoma cells. J Proteome Res 11(3):1609-1620. doi:10.1021/pr200790e

Buron N, Porceddu M, Brabant M et al (2010) Use of human cancer cell lines mitochondria to explore the mechanisms of $\mathrm{BH} 3$ peptides and ABT-737-induced mitochondrial membrane permeabilization. PLoS ONE 5(3):e9924. doi:10.1371/journal.pone.0009924

Castellino S, O'Mara M, Koch K, Borts DJ, Bowers GD, MacLauchlin C (2012) Human metabolism of lapatinib, a dual kinase inhibitor: implications for hepatotoxicity. Drug Metab Dispos 40(1):139-150. doi:10.1124/dmd.111.040949

Feng B, Xu JJ, Bi YA et al (2009) Role of hepatic transporters in the disposition and hepatotoxicity of a HER2 tyrosine kinase inhibitor CP-724,714. Toxicol Sci 108(2):492-500. doi:10.1093/ toxsci/kfp033

Gusdon AM, Fernandez-Bueno GA, Wohlgemuth S, Fernandez J, Chen J, Mathews CE (2015) Respiration and substrate transport rates as well as reactive oxygen species production distinguish mitochondria from brain and liver. BMC Biochem 16:22. doi:10.1186/s12858-015-0051-8

Kirby DM, Thorburn DR, Turnbull DM, Taylor RW (2007) Biochemical assays of respiratory chain complex activity. Methods Cell Biol 80:93-119. doi:10.1016/S0091-679X(06)80004-X

Kruger NJ (1994) The Bradford method for protein quantitation. Methods Mol Biol 32:9-15. doi:10.1385/0-89603-268-X:9

Mattiasson G (2004) Flow cytometric analysis of isolated liver mitochondria to detect changes relevant to cell death. Cytometry A 60(2):145-154. doi:10.1002/cyto.a.20024

McGill MR, Sharpe MR, Williams CD, Taha M, Curry SC, Jaeschke $\mathrm{H}$ (2012) The mechanism underlying acetaminophen-induced hepatotoxicity in humans and mice involves mitochondrial damage and nuclear DNA fragmentation. J Clin Invest 122(4):15741583. doi:10.1172/JCI59755

Meyers LL, Beierschmitt WP, Khairallah EA, Cohen SD (1988) Acetaminophen-induced inhibition of hepatic mitochondrial respiration in mice. Toxicol Appl Pharmacol 93(3):378-387

Miller TJ, Knapton A, Adeyemo O, Noory L, Weaver J, Hanig JP (2008) Cytochrome c: a non-invasive biomarker of drug-induced liver injury. J Appl Toxicol 28(7):815-828. doi:10.1002/jat.1347

Porceddu M, Buron N, Roussel C, Labbe G, Fromenty B, BorgneSanchez A (2012) Prediction of liver injury induced by chemicals in human with a multiparametric assay on isolated mouse liver mitochondria. Toxicol Sci 129(2):332-345. doi:10.1093/ toxsci/kfs 197

Rivkees SA (2010) 63 years and 715 days to the "boxed warning": unmasking of the propylthiouracil problem. Int J Pediatr Endocrinol. doi:10.1155/2010/658267

Shah F, Leung L, Barton HA et al (2015) Setting clinical exposure levels of concern for drug-induced liver injury (DILI) using mechanistic in vitro assays. Toxicol Sci 147(2):500-514. doi: $10.1093 /$ toxsci/kfv152

Shi Q, Yang X, Mattes WB, Mendrick DL, Harrill AH, Beger RD (2015) Circulating mitochondrial biomarkers for drug-induced liver injury. Biomark Med 9(11):1215-1223. doi:10.2217/ bmm. 15.59

Sugiyama E, Umemura S, Nomura $\mathrm{S}$ et al (2015) Impact of single nucleotide polymorphisms on severe hepatotoxicity induced by EGFR tyrosine kinase inhibitors in patients with non-small cell lung cancer harboring EGFR mutations. Lung Cancer 90(2):307-313. doi:10.1016/j.lungcan.2015.08.004

Takimoto T, Kijima T, Otani Y et al (2013) Polymorphisms of CYP2D6 gene and gefitinib-induced hepatotoxicity. Clin Lung Cancer 14(5):502-507. doi:10.1016/j.cllc.2013.03.003

Teo YL, Ho HK, Chan A (2015) Formation of reactive metabolites and management of tyrosine kinase inhibitor-induced hepatotoxicity: a literature review. Expert Opin Drug Metab Toxicol 11(2):231-242. doi:10.1517/17425255.2015.983075

Weng Z, Zhou P, Salminen WF et al (2014) Green tea epigallocatechin gallate binds to and inhibits respiratory complexes in swelling but not normal rat hepatic mitochondria. Biochem 
Biophys Res Commun 443(3):1097-1104. doi:10.1016/j. bbrc.2013.12.110

Weng Z, Luo Y, Yang X et al (2015a) Regorafenib impairs mitochondrial functions, activates AMP-activated protein kinase, induces autophagy, and causes rat hepatocyte necrosis. Toxicology 327:10-21. doi:10.1016/j.tox.2014.11.002

Weng Z, Wang K, Li H, Shi Q (2015b) A comprehensive study of the association between drug hepatotoxicity and daily dose, liver metabolism, and lipophilicity using 975 oral medications. Oncotarget 6(19):17031-17038. doi:10.18632/oncotarget.4400
Will Y, Dykens JA, Nadanaciva S et al (2008) Effect of the multitargeted tyrosine kinase inhibitors imatinib, dasatinib, sunitinib, and sorafenib on mitochondrial function in isolated rat heart mitochondria and H9c2 cells. Toxicol Sci 106(1):153-161. doi:10.1093/toxsci/kfn157

Xue T, Luo P, Zhu H et al (2012) Oxidative stress is involved in Dasatinib-induced apoptosis in rat primary hepatocytes. Toxicol Appl Pharmacol 261(3):280-291. doi:10.1016/j.taap.2012.04.010 